INSTITUTE OF FOOD AND RESOURCE ECONOMICS

UNIVERSITY OF COPENHAGEN

MSAP Working Paper Series

No. $06 / 2012$

Sharing the Cost of Redundant Items

Jens Leth Hougaard

Institute of Food and Resource Economics

University of Copenhagen

Hervé Moulin

Department of Economics

Rice University

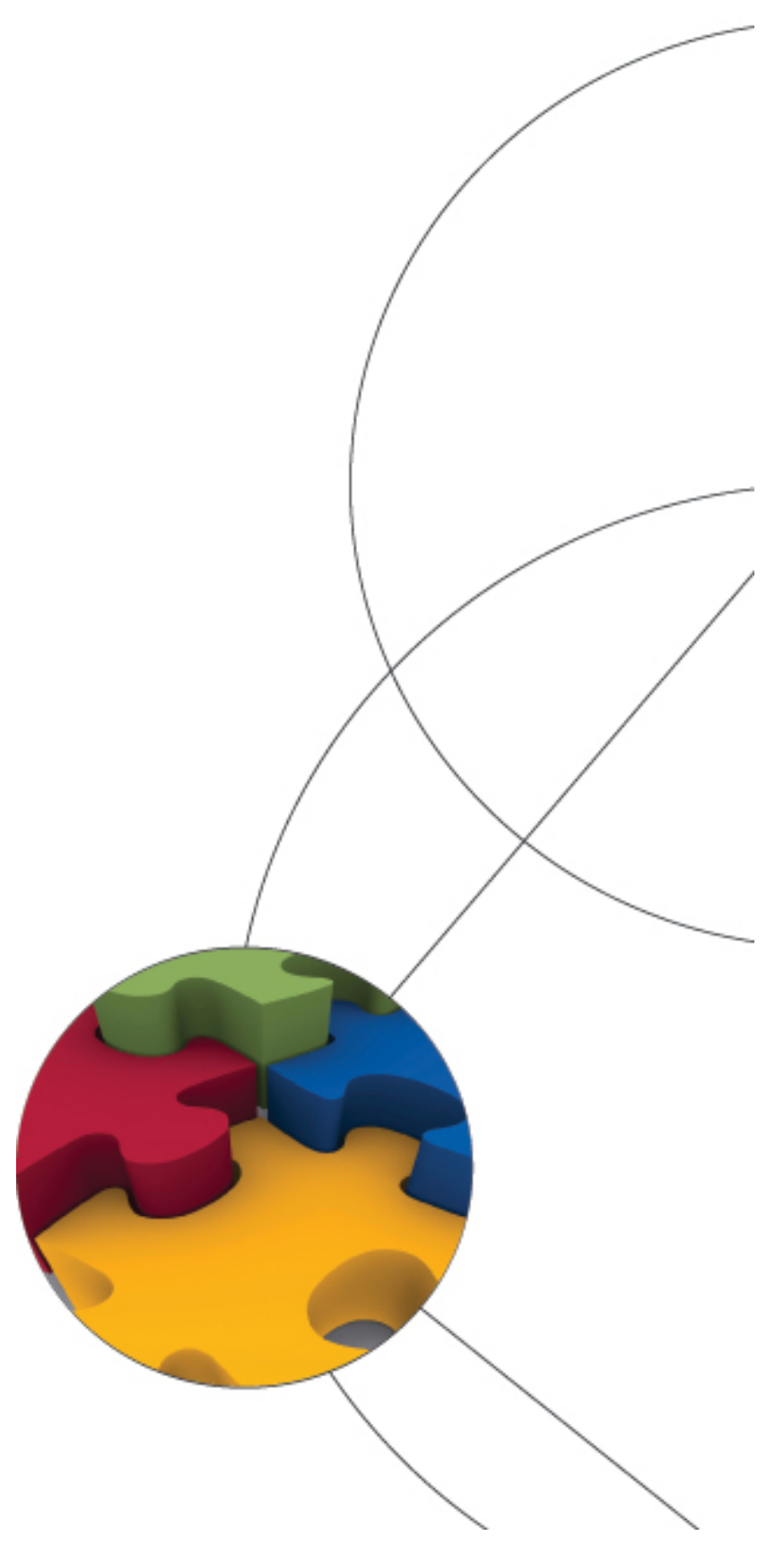




\title{
Sharing the Cost of Redundant Items
}

\section{Jens Leth Hougaard* Hervé Moulin ${ }^{\dagger}$}

September 2012

\begin{abstract}
We ask how to share the cost of finitely many public goods (items) among users with different needs: some smaller subsets of items are enough to serve the needs of each user, yet the cost of all items must be covered, even if this entails inefficiently paying for redundant items. Typical examples are network connectivity problems when an existing (possibly inefficient) network must be maintained.

We axiomatize a family of simple usage indices, one for each agent and for each item, measuring the relative worth of this item across agents, and generating cost sharing rules additive in costs.
\end{abstract}

Keywords: Cost sharing; Redundant costs; Connection networks; Connectivity

JEL Classification: C71, D30, D85, M41.

Acknowledgements: Hougaard acknowledges financial support from both the Danish Council for Independent Research (Social Sciences) and the Center for research in the Foundations of Electronic Markets (CFEM), funded by the Danish Council for Strategic Research. Moulin's research is supported by the National Science Foundation under Grant 1101202.

*Institute of Food and Resource Economics, University of Copenhagen, Rolighedsvej 25, DK-1958 Frederiksberg C., Denmark, phone +45 353368 14, email: jlh@foi.ku.dk

${ }^{\dagger}$ Department of Economics, Rice University, P.O.Box 1892, Houston, TX-77251, USA, phone +1 713348 4875, email: moulin@rice.edu 


\section{Introduction}

Consider a group of agents with different needs who must share the cost of several indivisible public goods (items). The needs of a user are met by certain combinations (subsets) of these goods, and the pattern of these subsets is arbitrary. This very general problem encompasses a variety of familiar fair division problems, including

- partners sharing a library of software licenses;

- cities sharing a set of antennas, routers, or other broadcasting devices geographically dispersed, so that each device reaches only a subset of cities;

- users of a network requiring certain connectivity between certain nodes of the graph, as in the minimal cost spanning tree problem, its variants where some agents require higher order connectivity, the traveling salesman problem, and more. ${ }^{1}$

What makes the problem difficult is that the substitutability of the different items can differ greatly across agents: out of three PC-software and three Macsoftware, one partner may need all three PC licenses, while another may be happy with any combination of two licenses, one of each type; or one network user may be served by any path connecting "his" node to the source, while another cannot use certain edges; and so on.

In contrast to essentially all the axiomatic cost sharing literature, we do not allow the agents to provide a cheapest (efficient) subset of items serving all individual needs. This is relevant to the construction of the network, or of the broadcasting devices, but it fails to address the equally important issue of sharing the cost of a given network. In our model the set of items is exogeneously fixed; it is enough to cover all needs, and typically include redundant items, i.e., items that can be discarded without any service interruption, but that our agents must nevertheless pay for. This assumption is realistic when the items represent long-lived pieces of infrastructure, such as antennas, edges of a physical utility network, or even licenses with multi-years subscription commitments: redesigning the set of items is not an option, and each item has usage and maintenance costs that must be covered (even closing or neutralizing an item can be costly). Therefore the familiar idea of assigning costs by focusing on the Stand Alone (efficient) cost of the various coalitions of agents plays almost no role in our analysis. ${ }^{2}$

An instance of our general model involves a fixed, finite, set $R$ of desirable "items", a specific cost for each item, and a set $N$ of users who must share the total cost of $R$. User $i$ has a set $\mathcal{D}^{i}$ of "service constraints" $S^{i}$ : providing all items in $S^{i}$ meets $i$ 's needs. For instance in connectivity games, the items are

\footnotetext{
${ }^{1}$ Moulin (2011) presents a recent review relating our model to the previous literature on cost sharing in networks.

${ }^{2}$ The only exception is the Lemma at the end of section 5 .
} 
edges of the network, and if a user needs to connect two specific nodes, $\mathcal{D}^{i}$ is the set of paths between these two nodes.

We propose and axiomatize a division of the total cost of all items based on an usage index for each item and each agent, capturing the importance of this item to this agent purely in terms of service options, independently of the cost of the item. Then we divide the cost of each item in proportion to the profile of usage indices: this ensures that individual shares are additive with respect to the profile of costs. Additivity with respect to costs is a familiar restriction on cost allocation rules, going back to Shapley's original axiomatization (Shapley, 1953), and maintained throughout most of the axiomatic cost sharing literature, see e.g., Moulin (2002). We adopt it for the usual reasons of computational simplicity and cost decentralization.

In the elementary case where items have no substitutability for any agent (each agent $i$ is served if and only if a certain set $S^{i}$ of items is provided: $\mathcal{D}^{i}=\left\{S^{i}\right\}$ ), agent $i$ 's usage index will be 1 for each item $a$ in $S^{i}$, and 0 for the items outside $S^{i}$. Here, our solutions divide the cost of each item equally between those agents who need it, or equally among all agents if this item is useless (is outside $\cup_{i} S^{i}$ ). An example of this situation is the classic "airport game" (Littlechild and Thompson, 1977), where this division coincides with the Shapley value of the corresponding Stand Alone cooperative game.

But the standard tools of cooperative game theory are not helpful for the interesting instances of our problem where the items are partally substitutable. Consider two agents, Ann and Bob, and two items $a, b$, such that Ann is served by either one of $a$ and $b$, while Bob needs both $a$ and $b$. Unlike Bob, Ann does not need a given item as she will be served by the other; accordingly she should pay less for each item because she is more flexible than Bob. The Stand Alone core requires Ann to pay at most the cost of the cheapest item. Combining this constraint with cost additivity forces Ann to pay nothing at all, as recommended in Moulin and Laigret (2011): Ann's free ride is clearly unfair to Bob since Ann still has access to be served. The Shapley value of the Stand Alone game is not additive in costs; it charges Ann one half of the cheapest item, say $a$ to fix ideas. Thus she is held as responsible as Bob for $a$, and not at all for $b$, just because $a$ happens to be cheaper than $b$, maybe by a very small amount. We submit that this is too hard on Ann with respect to $a$ (which she does not need as much as Bob), and too easy on her w.r.t. $b$ (which would be helpful to her if $a$ was unavailable).

Our counting index yields a more nuanced division of costs. For both items it assigns to Bob the maximal usage index 1, and a smaller yet non zero index to Ann. It is computed from the configuration of (inclusion) minimal serving sets for each agent: the usage index of an agent for a given item is the ratio of the number of his minimal serving sets containing the item, over the number of all his minimal serving sets. Here Ann has two minimal serving sets, $\{a\},\{b\}$, so her usage index is $1 / 2$ for both. Bob has only one minimal serving set $\{a, b\}$, so his usage index is 1 for both. Hence both costs are divided in proportions $1 / 3$ to Ann and $2 / 3$ to Bob, which we find more palatable in this example. 
Our main result in section 5, axiomatizes a one-dimensional family of usage indices, including the counting index just discussed, and the egalitarian index of 1 for all items in the union of minimal serving sets, $\cup_{\mathcal{D}^{i}} S^{i}$, and 0 outside this set. The central property in this result is the axiom "Irrelevance of Additional Needs" (IAN), stating that if a new item appears, which creates no new minimal service options for any agent, then the usage index (and thereby the cost allocation) of the original set of items remains unchanged. The IAN property is quite natural, and it has much bite. It allows for instance to "merge" two items that always appear together in the minimal serving sets of all agents: the single merged item inherits the common usage index of the two previous items.

\subsection{Relation to the literature}

The model was introduced by Moulin and Laigret (2011), who discuss the case where each item is indispensable to some agent: if we fail to provide any single item, someone is not served; they propose the equal-need solution, dividing the cost of any item equally between all agents who need this item for service. We saw in the above example with two items $a, b$, that this solution, charging everything to Bob, is too simplistic for our purpose. Moreover it relies on the Stand Alone core property, which may not be feasible in our model where items are typically redundant (not indispensable for any agent).

The set of serving sets of a given agent is a simple game where the items are the "players" (see e.g., Peleg and Südholter, 2003). Hence power indices (see e.g., Holler and Owen, 2001) of simple games could replace the usage indices we develop below in the proportional sharing of each item (see (2) below). Yet indices such as the Shapley value summing to 1 over all items, are not suited for our problem: by symmetry, in the above example with two items $a, b$, both Ann and Bob give the same index $\frac{1}{2}$ to each item, hence each cost is split equally, which ignores the fact that Ann is more flexible in her needs than Bob. Moreover, the Shapley value (and Banzhaf value for that matter) clearly violates the IAN property mentioned above.

\subsection{Contents}

We define the cost sharing problem in section 2, and usage indices in section 3 ; we introduce a handful of axiomatic properties in section 4 , and state and prove our main characterization Theorem in section 5. Section 6 illustrates our canonical counting method in a variety of examples, in particular in network connectivity problems. Some concluding comments are provided in section 7 .

\section{The model}

We fix the infinite sets $\mathcal{N}$ and $\mathcal{R}$ of potential agents and potential items (resources), respectively. The description of a problem entails, first, a finite set of agents $N, N \subset \mathcal{N}$, and a finite set of items $R, R \subset \mathcal{R}$. Next, the cost vector 
$c \in \mathbb{R}_{+}^{R}$ specifies the cost $c_{a}$ of each item $a \in R$. The items are public goods, consumed without rivalry by all agents in $N$, who must share the total cost $c_{R}=\sum_{a \in R} c_{a}$.

Finally, the needs of agent $i \in N$ are described by a non empty set $\mathcal{D}^{i} \subseteq$ $2^{R} \backslash \varnothing$ of (non empty) service constraints. That is, agent $i$ is served by any subset of items $S^{i} \in \mathcal{D}^{i}$ : if the items in $S^{i}$ are provided, other items are no longer useful to $i$. The sets $\mathcal{D}^{i}$ are inclusion monotonic: if $\mathcal{D}^{i}$ contains $S^{i}$ then it also contains any superset of $S^{i}$. This is the only restriction on service constraints.

A cost allocation problem is thus a list $\left(N, R,\left\{\mathcal{D}^{i}\right\}_{i \in N}, c\right)$. Denote by $\Gamma^{N, R}$ the set of cost allocation problems with agent set $N$ and item set $R$, and by $\Gamma$ the set of all allocation problems: $\Gamma=\bigcup_{N \in \mathcal{N}, R \in \mathcal{R}} \Gamma^{N, R}$.

Definition 1. A cost allocation rule $\psi$ is a mapping with domain $\Gamma$ such that $\psi\left(N, R,\left\{S^{i}\right\}_{i \in N}, c\right) \in \mathbb{R}_{+}^{N}$ for any problem in $\Gamma^{N, R}$, and moreover

$\sum_{i \in N} \psi_{i}\left(N, R,\left\{\mathcal{D}^{i}\right\}_{i \in N}, c\right)=c_{R}$.

\section{$3 \quad$ Usage indices}

The cost allocation rule $\psi$ is additive if, for any two problems that differ at most in their cost function, we have

$$
\psi\left(N, R,\left\{\mathcal{D}^{i}\right\}_{i \in N}, c+c^{\prime}\right)=\psi\left(N, R,\left\{\mathcal{D}^{i}\right\}_{i \in N}, c\right)+\psi\left(N, R,\left\{\mathcal{D}^{i}\right\}_{i \in N}, c^{\prime}\right)
$$

This amounts to write the rule $\psi$ as

$$
\psi\left(N, R,\left\{\mathcal{D}^{i}\right\}_{i \in N}, c\right)=\sum_{a \in R} c_{a} \times \varphi\left(N, R,\left\{\mathcal{D}^{i}\right\}_{i \in N}, a\right)
$$

where the vector $\varphi\left(N, R,\left\{\mathcal{D}^{i}\right\}_{i \in N}, a\right) \in \Delta(N)$ (the $N$-simplex) assigns relative shares in the division of $c_{a}$. We call its coordinate $\varphi_{j}\left(N, R,\left\{\mathcal{D}^{i}\right\}_{i \in N}, a\right)$ the usage index of resource $a$ by agent $j$, given the profile $\left\{\mathcal{D}^{i}\right\}_{i \in N}$ of service constraints.

Usage indices capture the relative importance of a given item for a given agent, relative to the full profile of service constraints. This interpretation suggests some natural restrictions on $\varphi$, when the need of a given agent for a given item is maximal, non zero, or null.

The need of agent $i$ for item $a$ is maximal when $a \in S$ for all $S \in \mathcal{D}^{i}$, in which case we say that item $a$ is indispensable to $i$. Let $G^{i}$ be the set of items indispensable to $i$. Fixing the service needs of agents other than $i$, we require that $i$ 's share of $a$ 's cost be largest when $a \in G^{i}$.

We say that for agent $i \in N$ the item $a \in R$ is critical at $S \in \mathcal{D}^{i}$ if $S \backslash a \notin \mathcal{D}^{i}$. By our assumption that $\mathcal{D}^{i}$ is made of non empty subsets of $R$, the set of critical items for agent $i, H^{i}=\left\{a \in R \mid a\right.$ is critical for some $\left.S \in \mathcal{D}^{i}\right\}$, is non empty (while in most of our examples, $G^{i}$ is empty for most $i$ ). An item critical for $i$ is of some use to this agent, so it is fair to hold $i$ responsible for some of its cost. On the contrary, if item $a$ is not critical for $i$, then providing $a$ has no impact 
on whether $i$ is served or not, so we do not charge him for $a$ 's cost. Finally, we require that the agents are equally responsible for a completely useless item.

Definition 2. A usage index associates to any $N \in \mathcal{N}, R \in \mathcal{R}$, and any profile of service constraints $\left.\left\{\mathcal{D}^{i}\right\}_{i \in N}, a\right)$, a vector of shares $\varphi\left(N, R,\left\{\mathcal{D}^{i}\right\}_{i \in N} ; a\right) \in$ $\Delta(N)$. The mapping $\varphi$ satisfies the following four assumptions:

i) if a is indispensable to $i$ at $\widetilde{\mathcal{D}}^{i}$, then $i$ 's cost share is largest:

$$
\varphi\left(\widetilde{\mathcal{D}}^{i},\left\{\mathcal{D}^{j}\right\}_{j \in N \backslash i} ; a\right) \geq \varphi\left(\mathcal{D}^{i},\left\{\mathcal{D}^{j}\right\}_{j \in N \backslash i} ; a\right) \text { for all } \mathcal{D}^{i} \subseteq 2^{R} \backslash \varnothing
$$

(where for simplicity we omit $N, R$ in the argument of $\varphi$ )

ii) if a is critical to $i$ at $\mathcal{D}^{i}$, then $i$ pays something of its cost:

$$
a \in H^{i} \Rightarrow \varphi_{i}\left(\left\{\mathcal{D}^{j}\right\}_{j \in N} ; a\right)>0
$$

iii) if a is not critical for $i$ at $\mathcal{D}^{i}$, but is critical to some other agent(s), then $i$ is not charged for $a$ :

$$
a \in\left\{R \backslash H^{i}\right\} \cap\left\{\cup_{j \in N \backslash\{i\}} H^{j}\right\} \Rightarrow \varphi_{i}\left(\left\{\mathcal{D}^{j}\right\}_{j \in N} ; a\right)=0
$$

$i v)$ if a is not critical for any agent, its cost is divided equally:

$$
a \in R \backslash \cup_{j \in N} H^{j} \Rightarrow \varphi_{i}\left(\left\{\mathcal{D}^{j}\right\}_{j \in N} ; a\right)=\frac{1}{|N|} \text { for all } i
$$

In the next section we introduce axiomatic properties powerful enough to characterize a one-dimensional family of usage indices. Indices in this family take the simple "proportional" form,

$$
\varphi_{i}\left(\left\{\mathcal{D}^{j}\right\}_{j \in N}, a\right)=\frac{\theta\left(\mathcal{D}^{i}, a\right)}{\sum_{j \in N} \theta\left(\mathcal{D}^{j}, a\right)} \text { for all } i,
$$

where $\theta\left(\mathcal{D}^{i}, a\right)$ is agent $i$ 's individual usage index for item $a$ at $\mathcal{D}^{i}$. This defines a usage index in the sense of Definition 2 if

$$
\begin{gathered}
\theta\left(\mathcal{D}^{i}, a\right) \geq 0 ; \theta\left(\mathcal{D}^{i}, a\right) \text { is maximal w.r.t. } \mathcal{D}^{i} \text { if } a \in G^{i} \\
\theta\left(\mathcal{D}^{i}, a\right)>0 \text { if } a \in H^{i} ; \theta\left(\mathcal{D}^{i}, a\right)=0 \text { if } a \notin H^{i}
\end{gathered}
$$

and we adopt the convention $\frac{0}{0}=\frac{1}{n}$

The simplest example of an individual usage index meeting (3) and (4), only checks whether an item is critical or not:

$$
\theta^{0}\left(\mathcal{D}^{i}, a\right)=1 \text { if } a \in H^{i}, \theta^{0}\left(\mathcal{D}^{i}, a\right)=0 \text { otherwise }
$$

This egalitarian index is very crude, ignoring much of the information conveyed by the serving sets. This is clear already for the two items example in the introduction: the corresponding rule splits all costs equally, whether with two or three items. Yet in large interconnected networks such as the Internet, we often see users paying flat fees, and service providers operating under peering 
agreements, despite substantial differences in size and required capacity: this suggests that $\theta^{0}$ is not unrealistic.

Note that property $i i$ ) in Definition 2 rules out the simple index ${ }^{3}$ which only charges the cost of item $a$ to agents for whom $a$ is indispensable:

$$
\theta^{G}\left(\mathcal{D}^{i}, a\right)=1 \text { if } a \in G^{i} ; \theta^{G}\left(\mathcal{D}^{i}, a\right)=0 \text { otherwise. }
$$

See however the first concluding comment (section 7) addressing the relaxation of property $i$ ).

Our next index focuses on the pattern of minimal serving sets for each agent $i$, i.e., on those serving sets for which all items are critical. Write $\overline{\mathcal{D}}^{i}=\{S \in$ $\left.\mathcal{D}^{i} \mid T \varsubsetneqq S \Rightarrow T \notin \mathcal{D}^{i}\right\}$ for the (non empty) set of minimal serving sets, and $\overline{\mathcal{D}}^{i}(a)=\left\{S \in \overline{\mathcal{D}}^{i} \mid a \in S\right\}$ for the subset of those containing item $a$. Note that $a \in G^{i}$ if and only if $\overline{\mathcal{D}}^{i}(a)=\overline{\mathcal{D}}^{i}$. We define the canonical counting index as follows,

$$
\theta^{1}\left(\mathcal{D}^{i}, a\right)=\frac{\left|\overline{\mathcal{D}}^{i}(a)\right|}{\left|\overline{\mathcal{D}}^{i}\right|}
$$

It clearly meets the properties (3) and (4). This index measures the centrality of a given item for the agent's service options. In connection networks this corresponds to the relative marginal connectivity of a given edge. Thus $\theta^{1}$ is reminiscent of the index of "betweenness centrality" describing, in the networks literature, the influence of given edges and nodes in a graph (see e.g., Jackson 2008).

Our last example is a probabilistic usage index. Given $\mathcal{D}^{i}$ and an ordering $\sigma: N \rightarrow[n]$ of $N$, let $\mathcal{F}^{i}(\sigma)$ be the set of minimal serving sets appearing first in this ordering,

$$
\mathcal{F}^{i}(\sigma)=\arg \min _{S^{i} \in \overline{\mathcal{D}}^{i}}\left\{\max _{a \in S^{i}} \sigma(a)\right\} .
$$

Moreover, let $\widetilde{S}^{i}(\sigma) \in \mathcal{F}^{i}(\sigma)$ be the set that lexicographically minimize the first coordinate in $\bar{S}^{i}$ given the order $\sigma$, and let $F^{i}=\cup_{\sigma} \widetilde{S}^{i}(\sigma)$. Now, define

$$
\theta^{p}\left(\mathcal{D}^{i}, a\right)=\frac{\left|\left\{\bar{S}^{i} \in F^{i}: a \in \bar{S}^{i}\right\}\right|}{|R| !}
$$

That is, $\theta^{p}\left(\mathcal{D}^{i}, a\right)$ is defined as the probability that $a \in F^{i}$, for a uniform random ordering of the items. In other words, it measures the "likelihood of usage" of an item $a$, in a hypothetical world where we only need to provide a minimal serving set. Clearly (3) and (4) hold true.

In the two-item example of the introduction, the indices $\theta^{p}$ and $\theta^{1}$ coincide ( 1 for Bob and $\frac{1}{2}$ for Ann, for each item). Add now a third item $d$, and assume

\footnotetext{
${ }^{3}$ In the subclass of problems $\left(N, R,\left\{\mathcal{D}^{i}\right\}_{i \in N}, c\right)$ such that each item is indispensable to someone $\left(R=\cup_{j \in N} G^{j}\right)$, the allocation rule derived from the index $\theta^{G}$ is characterized in Moulin and Laigret (2011) by the combination of Cost Additivity and the Stand Alone core property.
} 
that Ann's minimal serving sets are $\{a\},\{b\},\{d\}$, while Bob's are $\{a, b\},\{d\}$. Then we have

$$
\begin{gathered}
\theta^{1}(\text { Ann }, x)=\frac{1}{3} ; \theta^{1}(B o b, x)=\frac{1}{2}, \text { for } x=a, b, d \\
\theta^{p}(\text { Ann }, x)=\frac{1}{3} \text { for all } x ; \theta^{p}(B o b, x)=\frac{1}{3} \text { for } x=a, b, \theta^{p}(B o b, d)=\frac{2}{3}
\end{gathered}
$$

The probabilistic index is by design heavier on small minimal serving sets than on large ones, a normative choice which has merit but is not warranted by the primitives of the model: why should an agent be more responsible for items in small rather than large (minimal) serving sets? Example 2 in section 6.3 reinforces this point. The index $\theta^{p}$ is similarly sensitive to the merging of "twin" items, as we explain in the discussion of the IAN axiom below.

\section{Axioms for usage indices}

Our first two requirements are standard. A usage index should be oblivious to the name of agents: Anonymity, and to the labeling of items: Neutrality.

Anonymity (ANO): if $\sigma$ be a permutation of agents in $N$, then

$$
\left.\left.\varphi_{j}\left(\left\{\mathcal{D}^{i}\right\}_{i \in N}, a\right)\right)=\varphi_{\sigma(j)}\left(\left\{\mathcal{D}^{\sigma(i)}\right)\right\}_{i \in N}, a\right) \text { for all } j \in N
$$

Neutrality (NEU): if $\tau$ be a permutation of items in $R$, then

$$
\varphi\left(\left\{\mathcal{D}^{i}\right\}_{i \in N}, a\right)=\varphi\left(\left\{\tau\left(\mathcal{D}^{i}\right)\right\}_{i \in N}, \tau(a)\right)
$$

The next three properties are the heart of our proposal.

Consistency (CSY): for any $N \in \mathcal{N}, R \in \mathcal{R}, i \in N$, and $a \in R$, we have:

$\varphi_{-i}\left(N, R,\left\{\mathcal{D}^{j}\right\}_{j \in N}, a\right)=\left[1-\varphi_{i}\left(N, R,\left\{\mathcal{D}^{j}\right\}_{j \in N}, a\right)\right] \times \varphi\left(N \backslash\{i\}, R,\left\{\mathcal{D}^{j}\right\}_{j \in N \backslash i}, a\right)$

(both sides in $\mathbb{R}_{+}^{N \backslash\{i\}}$ )

Consistency is arguably one of the most popular requirements of allocation rules in the entire literature on fair division of resources and costs (surveyed in Thomson, 1998). In our model it states that upon removing an agent and reducing costs accordingly, the relative cost shares of the the remaining agents should be the same in the reduced and in the original problem.

Any usage index taking the simple proportional form (2), for some individual index $\theta\left(\mathcal{D}^{i}, a\right)$, is clearly anonymous and consistent; it is also neutral if $\theta$ is.

In the next axiom we fix $N \in \mathcal{N}, R \in \mathcal{R}$ and two problems $\left(N, R,\left\{\mathcal{D}^{j}\right\}_{j \in N}\right)$ and $\left(N, R^{\prime},\left\{\mathcal{D}^{\prime j}\right\}_{j \in N}\right)$ such that $R^{\prime}=R \cup\{b\}$ ( $b$ is outside $R$ ).

Irrelevance of Additional Needs (IAN): if for all $i \in N$ the service needs $\mathcal{D}^{i}$ and $\mathcal{D}^{\prime} i$ are such that:

$$
S \in \mathcal{D}^{\prime i} \Rightarrow S \backslash\{b\} \in \mathcal{D}^{i}
$$




$$
S \in \mathcal{D}^{i} \Rightarrow S \cup\{b\} \in \mathcal{D}^{\prime i}
$$

then $\varphi\left(R,\left\{\mathcal{D}^{i}\right\}_{i \in N}, a\right)=\varphi\left(R \cup\{b\},\left\{\mathcal{D}^{\prime i}\right\}_{i \in N}, a\right)$ for all $a \in R$.

Statements (8) and (9) mean that adding the new item $b$ may only create new serving sets of the form $S \cup\{b\}, S \in \mathcal{D}$. If $S$ is a minimal serving set in the old problem, $S \in \overline{\mathcal{D}}^{i}$, then either $S$ or $S \cup\{b\}$ is minimal in the new problem: indeed $S \cup\{b\}$ is in $\mathcal{D}^{\prime i}$ by (9), and by (8) no set $S^{\prime} \cup\{b\}$ where $S^{\prime} \varsubsetneqq S$ is serving $i$ in the new problem. Therefore there is a one-to-one correspondence between $\overline{\mathcal{D}}^{i}$ and $\overline{\mathcal{D}}^{\prime i}$, with some of the new sets including $b$. The IAN property says that this should not affect the usage index of any previous item. The index may change only when the addition of $b$ creates new service opportunities.

In the problem $\left(N, R,\left\{\mathcal{D}^{j}\right\}_{j \in N}\right)$ we say that items $a, b$, are twins if no agent needs one item and not the other:

$$
\text { for all } i \text { and all } S^{i} \in \overline{\mathcal{D}}^{i}:\left[\{a, b\} \subseteq S^{i}\right] \text { or }\left[\{a, b\} \cap S^{i}=\varnothing\right]
$$

A consequence of IAN is that if we remove a twin of item $a$, then the usage index of all other items, including $a$, are unchanged for all agents. Equivalently if we merge the two twin items in a single item, and add their costs, the profile $\psi\left(N, R,\left\{\mathcal{D}^{i}\right\}_{i \in N}, c\right)$ of individual cost shares is unchanged.

The axiom IAN is clearly satisfied by the egalitarian and canonical counting indices $\theta^{0}$ and $\theta^{1}$ above, but not by the probabilistic usage index $\theta^{p}$. Indeed the latter is not invariant to the merging operation just described. Compare the three-item problem (see end of section 3),

$$
\overline{\mathcal{D}}^{A n n}=\{\{a\},\{b\},\{d\}\} ; \overline{\mathcal{D}}^{B o b}=\{\{a, b\},\{d\}\}
$$

with the four-item problem,

$$
{\overline{\mathcal{D}^{\prime}}}^{A n n}=\left\{\left\{a, a^{\prime}\right\},\{b\},\{d\}\right\} ; \overline{\mathcal{D}}^{\prime B o b}=\left\{\left\{a, a^{\prime}, b\right\},\{d\}\right\}
$$

where $a$ and $a^{\prime}$ are twins. The counting index does not change:

$$
\theta^{1}(A n n, x)=\frac{1}{3} ; \theta^{1}(B o b, x)=\frac{1}{2}, \text { for } x=a, a^{\prime}, b, d
$$

but the probabilistic index becomes

$$
\begin{aligned}
& \theta^{p}(\text { Ann }, a)=\theta^{p}\left(\text { Ann }, a^{\prime}\right)=\frac{1}{6} ; \theta^{p}(\text { Ann }, b)=\theta^{p}(\text { Ann }, d)=\frac{5}{12} \\
& \theta^{p}(B o b, a)=\theta^{p}\left(B o b, a^{\prime}\right)=\theta^{p}(B o b, b)=\frac{1}{4} ; \theta^{p}(B o b, d)=\frac{3}{4}
\end{aligned}
$$

Our final axiom applies to $K$ isomorphic problems $\left(N, R_{k},\left\{\mathcal{D}_{k}^{j}\right\}_{j \in N}\right)$ : that is, the sets $R_{k}$ are pairwise disjoint and of the same size, and there is a bijection $\tau_{k k^{\prime}}$ from $R_{k}$ into $R_{k^{\prime}}$ sending $\mathcal{D}_{k}^{i}$ into $\mathcal{D}_{k^{\prime}}^{\prime i}$, and such that $\tau_{k^{\prime} k^{\prime}} \circ \tau_{k k^{\prime}}=\tau_{k k^{\prime}}$, for all $k, k^{\prime}, k "$. 
Replication (REP): in the problem $\left(N, \widetilde{R},\left\{\widetilde{\mathcal{D}}^{j}\right\}_{j \in N}\right)$, where $\widetilde{R}=\cup_{k} R_{k}$ and $\widetilde{\mathcal{D}}^{j}=\cup_{k} \mathcal{D}_{k}^{j}$, we have $\varphi\left(R_{1},\left\{\mathcal{D}^{j}\right\}_{j \in N}, a\right)=\varphi\left(\widetilde{R},\left\{\widetilde{\mathcal{D}}^{j}\right\}_{j \in N}, a\right)$ for all $a \in R_{1}$.

An agent is served in the $K$-replicated problem if and only if it is served in any one of the component problems. Think of the classical "airport game" where we must share the cost of several identical runways, while each airline's needs are covered by a single runway. Replication says that the cost of each runway will be split exactly as if the duplicate runways were absent.

Our three usage indices, egalitarian, counting, and probabilistic, all meet Replication.

\section{Characterization result(s)}

We consider a family of individual usage indices generalizing $\theta^{0}$ and $\theta^{1}$. For any number $\pi \geq 0$, define for any item $a$ and serving constraints $\mathcal{D}^{i}$ :

$$
\theta^{\pi}\left(\mathcal{D}^{i}, a\right)=\left(\frac{\left|\overline{\mathcal{D}}^{i}(a)\right|}{\left|\overline{\mathcal{D}}^{i}\right|}\right)^{\pi}
$$

and write $\varphi^{\pi}$ for the corresponding proportional usage index (2).

\section{Theorem:}

i) For all $\pi \geq 0$ the usage index $\varphi^{\pi}$ is anonymous, neutral, consistent, and meets Irrelevance of Additional Needs, and Replication.

ii) If the usage index $\varphi$ meets Anonymity, Neutrality, Consistency, Irrelevance of Additional Needs, and Replication, then $\varphi=\varphi^{\pi}$ for some $\pi \geq 0$, with the convention $(0)^{0}=0$.

\section{Proof.}

Step 1: The indices $\varphi^{\pi}$ are clearly anonymous and neutral. We check that $\varphi^{\pi}$ meets CSY. If $a \in \cup_{j \in N \backslash\{i\}} H^{j}$ the usage shares in the problems over $N, R$ and $N \backslash\{i\}, R$ take the form (2), thus equation (7) is a simple identity. If $a \in H^{i} \backslash\left\{\cup_{j \in N \backslash\{i\}} H^{j}\right\}$ the left-hand side of (7) is the null vector and $\varphi_{i}\left(N, R,\left\{\mathcal{D}^{j}\right\}_{j \in N}, a\right)=1$. Finally if $a \in R \backslash \cup_{j \in N} H^{j}$, equation (7) is the identity $\frac{1}{n}=\left(1-\frac{1}{n}\right) \frac{1}{n-1}$.

IAN holds because the bijection from $\overline{\mathcal{D}}^{i}$ into $\overline{\mathcal{D}}^{\prime i}$ is also from $\overline{\mathcal{D}}^{i}(a)$ into $\overline{\mathcal{D}}^{\prime i}(a)$, therefore $\frac{\left|\overline{\mathcal{D}}^{i}(a)\right|}{\left|\overline{\mathcal{D}}^{i}\right|}=\frac{\left|\overline{\mathcal{D}}^{\prime i}(a)\right|}{\left|\overline{\mathcal{D}}^{\prime i}\right|}$. Finally if we replicate the problem $\left(N, R_{1},\left\{\mathcal{D}^{j}\right\}_{j \in N}\right)$ to $\left(N, \widetilde{R},\left\{\widetilde{\mathcal{D}}^{j}\right\}_{j \in N}\right)$ as in the premises of REP, we get $\left|\widetilde{\widetilde{\mathcal{D}}}^{j}\right|=K \cdot\left|\overline{\mathcal{D}}^{j}\right|$ and $\left|\overline{\widetilde{\mathcal{D}}}^{j}(a)\right|=\left|\overline{\mathcal{D}}^{j}(a)\right|$ for all $j$, so that the ratio in (5) does not change.

We fix an usage index $\varphi$ meeting all our axioms, and show the converse statement in three steps. 
Step 2: Definition 2, Anonymity, and CSY, imply that $\varphi$ takes the proportional form (2), for an individual usage index $\theta\left(R, \mathcal{D}^{i}, a\right)$ meeting properties (3) and (4).

We fix throughout this step $R$, and an item $a$. Anonymity implies there exists for all $n$ a function $\xi^{n}\left(\mathcal{D} ; \mathcal{D}^{1}, \cdots, \mathcal{D}^{n-1}\right)$ defined on $\left(2^{2^{R} \backslash \varnothing}\right)^{n}$, symmetric in the $n-1$ variables $\mathcal{D}^{1}, \cdots, \mathcal{D}^{n-1}$, and such that $\varphi_{i}\left(\left\{\mathcal{D}^{j}\right\}_{j \in N}, a\right)=$ $\xi^{n}\left(\mathcal{D}^{i} ;\left\{\mathcal{D}^{j}\right\}_{j \in N \backslash\{i\}}\right)$ for all profiles of service needs.

Let $\mathcal{Z}$ be the subset of those serving constraints $\mathcal{D}$ in ${ }^{2^{R} \backslash \varnothing}$ such that $a$ is critical, $a \in H(\mathcal{D})$. Note that $|\mathcal{Z}| \geq 3$ for $|R| \geq 2$ (and for $|R|=1$ there is nothing to prove). By Definition $2, \xi^{3}\left(\mathcal{D}^{1} ; \mathcal{D}^{2}, \mathcal{D}^{3}\right)>0$ for all $\mathcal{D}^{1} ; \mathcal{D}^{2}, \mathcal{D}^{3}$ in $\mathcal{Z}$. Equation (7) applied twice with $N=\{1,2,3\}$ and $i=1,2$ gives,

$$
\frac{\xi^{3}\left(\mathcal{D}^{1} ; \mathcal{D}^{2}, \mathcal{D}^{3}\right)}{\xi^{3}\left(\mathcal{D}^{2} ; \mathcal{D}^{1}, \mathcal{D}^{3}\right)}=\frac{\xi^{2}\left(\mathcal{D}^{1} ; \mathcal{D}^{2}\right)}{\xi^{2}\left(\mathcal{D}^{2} ; \mathcal{D}^{1}\right)}
$$

Setting $\frac{\xi^{2}\left(\mathcal{D}^{1} ; \mathcal{D}^{2}\right)}{\xi^{2}\left(\mathcal{D}^{2} ; \mathcal{D}^{1}\right)}=\rho\left(\mathcal{D}^{1}, \mathcal{D}^{2}\right)$ (not symmetric in the two variables), and combining the above equality with two similar ones where the roles of the agents are permuted, we have, for all $\mathcal{D}^{1} ; \mathcal{D}^{2}, \mathcal{D}^{3}$ in $\mathcal{Z}$ :

$$
\rho\left(\mathcal{D}^{1}, \mathcal{D}^{2}\right) \times \rho\left(\mathcal{D}^{2}, \mathcal{D}^{3}\right) \times \rho\left(\mathcal{D}^{3}, \mathcal{D}^{1}\right)=1
$$

Therefore $\rho\left(\mathcal{D}^{1}, \mathcal{D}^{2}\right)=\frac{f\left(\mathcal{D}^{1}\right)}{g\left(\mathcal{D}^{2}\right)}$ for some positive functions $f, g$. We can take $f=g=\theta$ because $\rho\left(\mathcal{D}^{1}, \mathcal{D}^{2}\right) \times \rho\left(\mathcal{D}^{2}, \mathcal{D}^{1}\right)=1$, which implies

$$
\frac{\xi^{2}\left(\mathcal{D}^{1} ; \mathcal{D}^{2}\right)}{\theta\left(\mathcal{D}^{1}\right)}=\frac{\xi^{2}\left(\mathcal{D}^{2} ; \mathcal{D}^{1}\right)}{\theta\left(\mathcal{D}^{2}\right)} \Rightarrow \frac{\xi^{3}\left(\mathcal{D}^{1} ; \mathcal{D}^{2}, \mathcal{D}^{3}\right)}{\theta\left(\mathcal{D}^{1}\right)}=\frac{\xi^{3}\left(\mathcal{D}^{2} ; \mathcal{D}^{1}, \mathcal{D}^{3}\right)}{\theta\left(\mathcal{D}^{2}\right)}
$$

Repeated applications of CSY give

$$
\frac{\xi^{n}\left(\mathcal{D}^{1} ;\left\{\mathcal{D}^{j}\right\}_{j=2, \cdots, n}\right)}{\theta\left(\mathcal{D}^{1}\right)}=\frac{\xi^{n}\left(\mathcal{D}^{2} ;\left\{\mathcal{D}^{j}\right\}_{j=1,3, \cdots, n}\right)}{\theta\left(\mathcal{D}^{2}\right)}
$$

for all $n$ and all profiles in $\mathcal{Z}$. Therefore $\varphi$ takes the proportional form (2) whenever all $\mathcal{D}^{j}$ are in $\mathcal{Z}$. We set $\theta(\mathcal{D})=0$ if $\mathcal{D} \notin \mathcal{Z}$, and it is now clear that the index $\varphi$ defined by (2), and the convention $\frac{0}{0}=\frac{1}{n}$ is an usage index in the sense of Definition 2.

Step 3: Irrelevance of Additional Needs implies the form

$$
\theta(R, \mathcal{D}, a)=g(|\overline{\mathcal{D}}|,|\overline{\mathcal{D}}(a)|) \text { for all } R, \mathcal{D}, a
$$

where $g(p, q)$ is defined for all integers $p, q$ such that $0 \leq q \leq p \geq 1, g(p, q)>0$ if $q \geq 1$ and $g(p, q)=0$ if $q=0$.

The canonical mapping from an arbitrary service constraint $\mathcal{D}$ to its inclusion minimal subsets $\overline{\mathcal{D}}$ is a bijection between the set of service constraints, and that of minimal service constraints. Hence we can write $\theta(R, \overline{\mathcal{D}}, a)$ instead of $\theta(R, \mathcal{D}, a)$. Fix $a$, consider a problem with two agents $\left(R,\left\{\mathcal{D}^{i}\right\}_{i=1,2}\right)$, and set 
$p_{i}=\left|\overline{\mathcal{D}}^{i}\right|, q_{i}=\left|\overline{\mathcal{D}}^{i}(a)\right|$. Assume $q_{2} \geq 1$ ( $a$ is critical). We choose a set $X$ labeled $X=\{1,2, \cdots\}$ of proxy items, disjoint from $R$ and with cardinality $p_{1}+p_{2}$. We add item 1 in $X$ to the first serving set in $\overline{\mathcal{D}}^{1}(a)$, and nowhere else: this addition satisfies the premises of IAN thus it respects the shares of item $a$. Next we add item 2 in $X$ to the second serving set in $\overline{\mathcal{D}}^{1}(a)$, and nowhere else: again the the shares of item $a$ do not change. We continue adding one different proxy item to exactly one minimal serving set of a single agent, starting with the serving sets in $\overline{\mathcal{D}}^{1}(a)$ and continuing with those in $\overline{\mathcal{D}}^{1} \backslash \overline{\mathcal{D}}^{1}(a)$. Thus after the first $p_{1}$ items augment each element of $\overline{\mathcal{D}}^{1}$, we add item $p_{1}+1$ to the first set in $\overline{\mathcal{D}}^{2}(a)$, and so on. After we have placed all items in $X$, each set $S \in \overline{\mathcal{D}}^{i}, i=1,2$, is augmented by its personal proxy item, and shares of $a$ are still as in the original problem $\left(R,\left\{\mathcal{D}^{i}\right\}_{i=1,2}\right)$. Next we remove, one at a time, all items in $R \backslash a$, keeping the proxy items fixed. IAN again implies that the shares of $a$ do not change. The final problem we reach has the items $X \cup\{a\}$, and the minimal serving sets $\mathcal{T}^{i}$ such that

$$
\begin{gathered}
\overline{\mathcal{T}}^{1}=\left\{\{a, 1\},\{a, 2\}, \cdots,\left\{a, q_{1}\right\},\left\{q_{1}+1\right\},\left\{q_{1}+2\right\}, \cdots,\left\{p_{1}\right\}\right\} ; \\
\overline{\mathcal{T}}^{2}=\left\{\left\{a,\left(p_{1}+1\right)\right\}, \cdots,\left\{a,\left(p_{1}+q_{2}\right)\right\},\left\{p_{1}+q_{2}+1\right\}, \cdots,\left\{p_{1}+p_{2}\right\}\right\}
\end{gathered}
$$

The ratio $\frac{\theta\left(X \cup\{a\}, \overline{\mathcal{T}}^{1}, a\right)}{\theta\left(X \cup\{a\}, \overline{\mathcal{T}}^{2}, a\right)}$ is well defined because we assumed $\overline{\mathcal{D}}^{2}(a)$ is non empty. By Neutrality it depends only upon $|X \cup\{a\}|=1+p_{1}+p_{2}, p_{i}$ and $q_{i}$ for $i=1,2$. The construction above ensures that this ratio is the same as $\frac{\theta\left(R, \overline{\mathcal{D}}^{1}, a\right)}{\theta\left(R, \overline{\mathcal{D}}^{2}, a\right)}$, so we can write

$$
\frac{\theta\left(R, \overline{\mathcal{D}}^{1}, a\right)}{\theta\left(R, \overline{\mathcal{D}}^{2}, a\right)}=f\left(p_{1}, q_{1}, p_{2}, q_{2}\right)
$$

for some function $f$. Fixing $p_{2}=q_{2}=1$ (i.e., all items are indispensable to agent 2), we get $\theta\left(R, \overline{\mathcal{D}}^{1}, a\right)=h(|R|) \times f\left(p_{1}, q_{1}\right)$. We can clearly drop the term $h(|R|)$ that plays no role in the proportional form (2), so the proof of step 3 is complete.

Step 4. Replication implies $\varphi=\varphi^{\pi}$ for some $\pi \geq 0$.

Steps 2 and 3 imply the following form for our index:

$$
\varphi_{i}\left(N, R,\left\{\mathcal{D}^{j}\right\}_{j \in N}, a\right)=\frac{g\left(\left|\overline{\mathcal{D}}^{i}\right|,\left|\overline{\mathcal{D}}^{i}(a)\right|\right)}{\sum_{N} g\left(\left|\overline{\mathcal{D}}^{j}\right|,\left|\overline{\mathcal{D}}^{j}(a)\right|\right)}
$$

for all problems of all sizes.

Property $i)$ in Definition 2 says that $\varphi_{i}\left(N, R,\left\{\mathcal{D}^{i}\right\}_{i \in N}, a\right)$ is largest w.r.t. $\mathcal{D}^{i}$ when $\overline{\mathcal{D}}^{i}(a)=\overline{\mathcal{D}}^{i}$. This amounts to $g(p, p) \geq g\left(p^{\prime}, q^{\prime}\right)$ whenever these cardinalities are jointly feasible for some $R$. If $|R|=r$ then all $(p, p)$ s.t. $p \leq t-1$ are feasible; as $R$ can be arbitrarily large, we conclude $g(p, p)=g\left(p^{\prime}, p^{\prime}\right)$ for all $p, p^{\prime} \geq 1$. Without loss of generality we set $g(p, p)=1$. Thus

$$
0 \leq g(p, q) \leq 1 \text { for all } 0 \leq q \leq p \geq 1
$$


with left-equality if and only if $q=0$, and right-equality if $p=q$.

Apply now Replication to $K$ isomorphic problems $\left(N, R_{k},\left\{\mathcal{D}_{k}^{j}\right\}_{j=1,2}\right)$ as in the premises of REP, and to $a \in R_{1}$, critical for $\mathcal{D}_{1}^{1}$ and $\mathcal{D}_{1}^{2}$ :

$$
\frac{g\left(p_{i}, q_{i}\right)}{\sum_{1,2} g\left(p_{j}, q_{j}\right)}=\frac{g\left(K p_{i}, q_{i}\right)}{\sum_{1,2} g\left(K p_{j}, q_{j}\right)} \Leftrightarrow \frac{g\left(p_{1}, q_{1}\right)}{g\left(p_{2}, q_{2}\right)}=\frac{g\left(K p_{1}, q_{1}\right)}{g\left(K p_{2}, q_{2}\right)}
$$

for any $p_{i}, q_{i}$, with $q_{2} \geq 1$. Taking $p_{2}=q_{2}=1$ gives $g\left(K p_{1}, q_{1}\right)=g(K, 1) g\left(p_{1}, q_{1}\right)$, and renaming variables we have

$$
g\left(p p^{\prime}, q\right)=g\left(p^{\prime}, 1\right) g(p, q) \text { for all } 1 \leq q \leq p \text { and } 1 \leq p^{\prime}
$$

Exchanging the role of $p, p^{\prime}$ gives

$$
\begin{gathered}
g\left(p^{\prime}, 1\right) g(p, q)=g(p, 1) g\left(p^{\prime}, q\right) \text { for all } 1 \leq q \leq p, p^{\prime} \\
\Rightarrow \frac{g(p, q)}{g(p, 1)}=\frac{g\left(p^{\prime}, q\right)}{g\left(p^{\prime}, 1\right)}=f(q)
\end{gathered}
$$

for a function $f$, such that $f(0)=0$ and $f(q)>0$ for $q \geq 1$. From $g(p, p)=1$, we get $g(p, q)=\frac{f(q)}{f(p)}$. Plugging this in equation (13) gives $f\left(p p^{\prime}\right)=\frac{1}{f(1)} f(p) f\left(p^{\prime}\right)$ for all $p, p^{\prime} \geq 1$, so after one more innocuous normalization $f(1)=1$, we conclude that $f$ is multiplicative:

$$
f(p q)=f(p) f(q) \text { for all } p, q \geq 0 ; f(0)=0 ; f(q)>0 \text { for } q \geq 1
$$

Moreover $g(p, q) \leq 1$ implies that $f$ is weakly increasing.

It remains to prove that $f$ is a power function with non negative exponent. Fix two integers $p, q \geq 1$. For any $x, y, x^{\prime}, y^{\prime} \in \mathbb{N}$ the monotonicity and multiplicative character of $f$ imply

$$
p^{x} q^{y} \leq p^{x^{\prime}} q^{y^{\prime}} \Rightarrow f(p)^{x} f(q)^{y} \leq f(p)^{x^{\prime}} f(q)^{y^{\prime}}
$$

i.e., in $\log$ form

$$
\left(x-x^{\prime}\right) \ln p \leq\left(y^{\prime}-y\right) \ln q \Rightarrow\left(x-x^{\prime}\right) \ln f(p) \leq\left(y^{\prime}-y\right) \ln f(q)
$$

As $x, y, x^{\prime}, y^{\prime}$ are arbitrary, this implies that the vectors $(\ln p, \ln q)$ and $(\ln f(p), \ln f(q))$ are collinear. Taking $q=1$ we get $\ln f(p)>0$ for $p \geq 2$; taking $p, q \geq 2$ we see that $\frac{\ln f(p)}{\ln p}$ is constant for $p \geq 2$, thus there is a constant $\pi$ such that $\ln f(p)=\pi \ln p$ for all $p$, including $p=1$ because $f(1)=1$. The monotonicity of $f$ implies $\pi \geq 0$, hence $g(p, q)$ takes the desired form $g(p, q)=\left(\frac{q}{p}\right)^{\pi}$, and the proof of step 4 is complete.

The additive cost allocation rule derived from the index $\varphi^{\pi}$ is

$$
\psi_{i}^{\pi}\left(N, R,\left\{\mathcal{D}^{j}\right\}_{j \in N}, c\right)=\sum_{a \in R} \frac{\left(\frac{\left|\overline{\mathcal{D}}^{i}(a)\right|}{\left|\overline{\mathcal{D}}^{i}\right|}\right)^{\pi}}{\sum_{N}\left(\frac{\left|\overline{\mathcal{D}}^{j}(a)\right|}{\left|\overline{\mathcal{D}}^{j}\right|}\right)^{\pi}} c_{a} \text {, for all } i .
$$


For $\pi=0$, the rule $\psi^{0}$ divides the cost of item $a$ equally among all agents for whom $a$ is critical, or equally among all agents if $a$ is critical for nobody. This is where our convention $(0)^{0}=0$ plays a role.

For $\pi=1$ the individual usage index $\theta^{1}$ is the canonical counting index (5); we call the corresponding rule the counting rule:

$$
\psi_{i}^{1}\left(N, R,\left\{\mathcal{D}^{j}\right\}_{j \in N}, c\right)=\sum_{a \in R} \frac{\frac{\left|\overline{\mathcal{D}}^{i}(a)\right|}{\left|\overline{\mathcal{D}}^{i}\right|}}{\sum_{N} \frac{\left|\overline{\mathcal{D}}^{j}(a)\right|}{\left|\overline{\mathcal{D}}^{j}\right|}} c_{a}, \text { for all } i
$$

The Theorem implies a characterization of the family of rules (14) by means of the same five axioms (suitably reformulated in terms of cost allocation rules), plus cost additivity. We omit the details.

More interesting is the fact that if $\pi \in[0,1]$, and only then, the cost allocation rule $\psi^{\pi}$ satisfies a natural lower bound on cost shares. In a problem $\left(N, R,\left\{\mathcal{D}^{j}\right\}_{j \in N}, c\right)$ the Stand Alone cost of agent $i$ is: $s a\left(\mathcal{D}^{i}, c\right)=\min _{S \in \mathcal{D}^{i}} c_{S}$. The unanimity cost share is $\frac{1}{n} s a\left(\mathcal{D}^{i}, c\right)$ : before we know anything about the other agents' characteristics, we can hold agent $i$ responsible for at least her fair share of her stand alone cost:

Unanimity Lower bound: for all problems $\left(N, R,\left\{\mathcal{D}^{j}\right\}_{j \in N}, c\right)$ and all $i$ :

$$
\psi_{i}\left(\left\{\mathcal{D}^{j}\right\}_{j \in N}, c\right) \geq \frac{1}{n} s a\left(\mathcal{D}^{i}, c\right)
$$

Lemma: The cost allocation rule $\psi^{\pi}$ in (14) satisfies the Unanimity Lower Bound if and only if $\pi \in[0,1]$.

Proof. If statement. For any numbers $x_{i}, i \in N$ and $\pi$, all in $[0,1]$, we have

$$
\frac{x_{i}^{\pi}}{\sum_{N} x_{j}^{\pi}} \geq \frac{x_{i}^{\pi}}{(n-1)+x_{i}^{\pi}} \geq \frac{1}{n} x_{i}
$$

Fix a problem $\left(N, R,\left\{\mathcal{D}^{j}\right\}_{j \in N}, c\right)$ and apply this to $x_{i}=\frac{\left|\overline{\mathcal{D}}^{i}(a)\right|}{\left|\overline{\mathcal{D}}^{i}\right|}$ :

$$
\begin{gathered}
\psi_{i}^{\pi}\left(N, R,\left\{\mathcal{D}^{j}\right\}_{j \in N}, c\right)=\sum_{a \in R} \frac{x_{i}^{\pi}}{\sum_{N} x_{j}^{\pi}} c_{a} \geq \frac{1}{n} \sum_{a \in R} \frac{\left|\overline{\mathcal{D}}^{i}(a)\right|}{\left|\overline{\mathcal{D}}^{i}\right|} c_{a} \\
=\frac{1}{n\left|\overline{\mathcal{D}}^{i}\right|} \sum_{a \in R}\left|\overline{\mathcal{D}}^{i}(a)\right| c_{a}=\frac{1}{n}\left\{\frac{1}{\left|\overline{\mathcal{D}}^{i}\right|} \sum_{S \in \overline{\mathcal{D}}^{i}} c_{S}\right\}
\end{gathered}
$$

from which the desired conclusion follows because $c_{S} \geq s a\left(\mathcal{D}^{i}, c\right)$ for each $S \in$ $\overline{\mathcal{D}}^{i}$.

Only if statement. Fix $\pi>1$. Pick an arbitrary set of items $R$, and an agent $i$ with service constraints $\mathcal{D}^{i}=(\{a\}, a \in R)$, so $\frac{\left|\overline{\mathcal{D}}^{i}(a)\right|}{\left|\overline{\mathcal{D}}^{i}\right|}=\frac{1}{|R|}$ for all $a$. Complete 
the population $N$ with $n-1$ identical agents s.t. $\frac{\left|\overline{\mathcal{D}}^{j}(a)\right|}{\left|\overline{\mathcal{D}}^{j}\right|}=1$, and set $c_{a}=1$ for all $a$. Then $s a\left(\mathcal{D}^{i}, c\right)=1$, while

$$
\psi_{i}^{\pi}\left(N, R,\left\{\mathcal{D}^{j}\right\}_{j \in N}, c\right)=|R| \frac{\frac{1}{|R|^{\pi}}}{n-1+\frac{1}{|R|^{\pi}}}
$$

Setting $x=\frac{1}{|R|}$, we have

$$
\psi_{i}^{\pi}\left(\left\{\mathcal{D}^{j}\right\}_{j \in N}, c\right)<\frac{1}{n} \Leftrightarrow n\left(x-x^{\pi}\right)+x^{\pi-1}>x
$$

and this inequality holds for $n$ large enough because $x-x^{\pi}>0$.

Note that the rule in proportional form (2) satisfies the Unanimity Lower Bound as well when the index $\theta$ is the probabilistic index (6).

We conclude the section by checking the independence of the axioms in our Theorem.

1) Drop Anonymity: fix a mapping $\mathcal{N} \ni i \rightarrow \lambda_{i}>0$; then the proportional usage index (2) with individual index

$$
\theta\left(\mathcal{D}^{i}, a\right)=\lambda_{i} \theta^{\pi}\left(\mathcal{D}^{i}, a\right)
$$

meets all other axioms.

2) Drop Neutrality: fix a mapping $\mathcal{R} \ni a \rightarrow \pi_{a} \geq 0$; then the proportional usage index (2) with individual index

$$
\theta\left(\mathcal{D}^{i}, a\right)=\theta^{\pi_{a}}\left(\mathcal{D}^{i}, a\right)
$$

meets all other axioms.

3) Drop Consistency: define $M\left(\left\{\mathcal{D}^{j}\right\}_{j \in N}, a\right)=\arg \max _{i} \frac{\left|\overline{\mathcal{D}}^{i}(a)\right|}{\left|\overline{\mathcal{D}}^{i}\right|}$; then the usage index

$$
\begin{aligned}
\varphi_{i}\left(\left\{\mathcal{D}^{j}\right\}_{j \in N}, a\right) & =\frac{1}{2 n} & \text { if } i \notin M\left(\left\{\mathcal{D}^{j}\right\}_{j \in N}, a\right) \\
& =\frac{1}{2 n}+\frac{1}{2\left|M\left(\left\{\mathcal{D}^{j}\right\}_{j \in N}, a\right)\right|} & \text { if } i \in M\left(\left\{\mathcal{D}^{j}\right\}_{j \in N}, a\right)
\end{aligned}
$$

meets all other axioms.

4) Drop Irrelevance of Additional Needs: then the proportional usage index (2) derived from the probabilistic index (6) meets all other axioms.

5) Drop Replication: choose an increasing homeomorphism $h$ from $[0,1]$ into itself that is not a power function; then the proportional usage index (2) with individual index

$$
\theta\left(\mathcal{D}^{i}, a\right)=h\left(\frac{\left|\overline{\mathcal{D}}^{i}(a)\right|}{\left|\overline{\mathcal{D}}^{i}\right|}\right)
$$

meets all other axioms. 


\section{Examples}

We present a series of examples displaying both good and bad features of our canonical counting method (15), in particular by comparing it to the probabilistic index. It is a solution to a very general class of problems so it is not surprising that in some specific situations the method produces questionable results.

\subsection{Set cover}

This is the case where for every agent, each minimal serving set is a singleton. So an item $a$ is a non empty subset of $N$, and providing $a$ meets the needs of all its members. The set $R$ is a subset of $2^{N} \backslash \varnothing$. The classic set cover problem (see e.g., Wolsey, 1998) looks for a cheapest set of items covering $N$. In our model we set $T^{i}=\{a \in R \mid i \in a\}$ and we have

$$
\mathcal{D}^{i}=\left\{S \subseteq R \mid S \cap T^{i} \neq \varnothing\right\} ; \overline{\mathcal{D}}^{i}=\left\{a \mid a \in T^{i}\right\} ; \overline{\mathcal{D}}^{i}(a)=\{a\}
$$

Therefore

$$
\theta^{1}\left(\mathcal{D}^{i}, a\right)=\frac{1}{\left|T^{i}\right|} \text { if } a \in T^{i} ; \theta^{1}\left(\mathcal{D}^{i}, a\right)=0 \text { otherwise }
$$

and the cost of $a$ is divided among all agents $i$ of $a$ in proportion to $\frac{1}{\left|T^{i}\right|}$.

The probabilistic usage index $\theta^{p}$ coincides with $\theta^{1}$ for the set cover problems.

\subsection{Homogenous items}

Example 1: identical items. Write $R(p)$ for the set of subsets of $R$ with $p$ elements. We assume that all items are identical, and agent $i$ needs at least $p_{i}$ items to be provided. Thus $\overline{\mathcal{D}}^{i}=R\left(p_{i}\right)$ and $\overline{\mathcal{D}}^{i}(a)$ selects those elements of $R(p)$ containing $a$. Hence

$$
\theta^{\pi}\left(p_{i}, a\right)=\left(\frac{\left(\begin{array}{c}
|R|-1 \\
p_{i}-1
\end{array}\right)}{\left(\begin{array}{c}
|R| \\
p_{i}
\end{array}\right)}\right)^{\pi}=\left(\frac{p_{i}}{|R|}\right)^{\pi}
$$

The counting method $\psi^{1}$ defined in (15) divides costs in proportion to the parameters $p_{i}$, a plausible recommendation which coincides, again, with that of the probabilistic usage index.

Example 2.1: two types of items. The counting methods may also result in less intuitive solutions. We now have 10 red items and 10 blue items. Ann needs any two items, Bob needs any three blue items or any red item. The canonical counting indices are

$$
\theta_{A n n}^{1}(b l u e)=\theta_{A n n}^{1}(\text { red })=\frac{1}{10}
$$




$$
\theta_{\text {Bob }}^{1}(\text { blue })=\frac{36}{130}=0.28 ; \theta_{\text {Bob }}^{1}(\text { red })=\frac{1}{130}=0.0076
$$

Thus Bob bears a much heavier responsibility for a blue item than for a red one, which is paradoxical because a red item is more useful to Bob than a blue one.

Assuming all items cost 1 , the cost shares are:

$\rightarrow$ for blue items: Ann $\$ 2.65$, Bob $\$ 7.35$

$\rightarrow$ for red items: Ann $\$ 9.29$, Bob $\$ 0.71$

The probabilistic usage index $\theta^{p}$ seems more realistic in this example:

$$
\theta_{\text {Bob }}^{p}(\text { blue })=\frac{3}{95}=0.032 ; \theta_{\text {Bob }}^{p}(\text { red })=\frac{17}{190}=0.089
$$

while $\theta_{A n n}^{p}$ remains 0.1 for all items. The corresponding cost shares are radically different:

$\rightarrow$ for blue items: Ann $\$ 7.6$, Bob $\$ 2.4$

$\rightarrow$ for red items: Ann $\$ 5.3$, Bob $\$ 4.7$

Note that the total bill of the two agents in the two rules are fairly close though.

\subsection{Network connections}

Example 1: several sources on a tree or a loop. The tree in the figure below represents a network where the three leaf nodes $\omega, \omega$, $\omega$ ", are "sources", while agents live on the other nodes. Each agent is served by a connection to any source node, and the costly items are the edges of the graph.

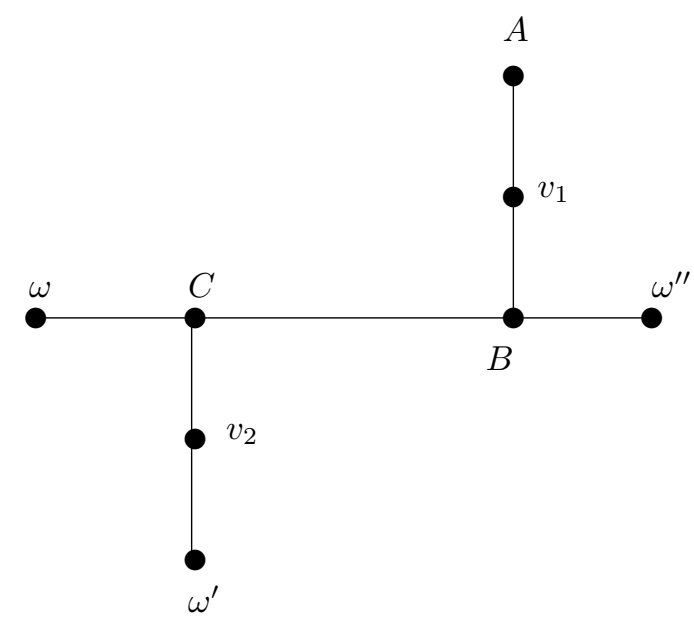

Write $\left[v, v^{\prime}\right]$ for the set of edges in the path from node $v$ to node $v^{\prime}$. Then for agent $i$ located at $v_{i}$, the minimal serving sets are

$$
\overline{\mathcal{D}}^{i}=\left\{\left[v_{i}, \omega\right],\left[v_{i}, \omega^{\prime}\right],\left[v_{i}, \omega^{\prime \prime}\right]\right\}
$$


Therefore the counting index of the agent located at $v_{1}$ is zero on $\left[v_{1}, A\right]$ and

$$
\theta^{1}\left(\mathcal{D}^{1}\right)=1 \text { on }\left[v_{1}, B\right],=\frac{1}{3} \text { on }[B, \omega "] \cup[C, \omega] \cup[C, \omega \prime],=\frac{2}{3} \text { on }[C, B]
$$

That of agent $v_{2}$ is zero on $[B A]$ and

$$
\theta^{1}\left(\mathcal{D}^{2}\right)=\frac{1}{3} \text { on }\left[v_{2}, \omega^{\prime}\right],=\frac{2}{3} \text { on }\left[v_{2}, C\right],=\frac{1}{3} \text { on }\left[C, \omega^{\prime \prime}\right] \text { and on }[C, \omega]
$$

It is easy to generalize these examples to an arbitrary tree with an arbitrary number of sources on the leafs of the tree. ${ }^{4}$

The probabilistic index is much harder to compute, as it depends upon the number of edges on the various paths like $\left[v_{1}, B\right],[B, \omega "]$, etc..The counting method recognizes that all edges in such paths are twins for agent 1 , and treats them as a single item.

The graph below contains a single loop and three leaf-sources $\omega, \omega^{\prime}, \omega^{\prime \prime}$, as before. Our counting index can easily handle problems where some agents require 1-connectivity to some source, while others need 2-connectivity: two edge disjoint paths from the agent to some sources.

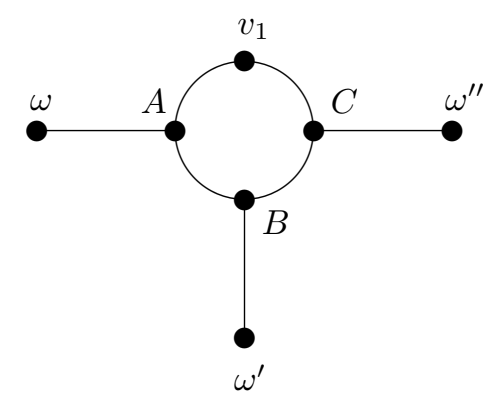

For agent 1 located at $v_{1}$ and seeking 1-connectivity, we have six minimal serving sets, i.e., the following paths

$$
\begin{aligned}
\overline{\mathcal{D}}^{1}= & \left\{v_{1} A \omega, v_{1} C B A \omega, v_{1} A B \omega^{\prime}, v_{1} C B \omega^{\prime}, v_{1} C \omega^{\prime \prime}, v_{1} A B C \omega^{\prime \prime}\right\} \\
& \Rightarrow \theta^{1}\left(\mathcal{D}^{1}\right)=\frac{1}{2} \text { on the loop, }=\frac{1}{3} \text { outside the loop }
\end{aligned}
$$

${ }^{4}$ If $\Omega$ is the set of sources, then the index of an edge $e$ is

$$
\theta^{1}(i ; e)=\frac{\left|\left\{\omega \in \Omega \mid e \in\left[\omega, v_{i}\right]\right\}\right|}{|\Omega|}
$$

The case where sources are not necessarily on leafs is almost as simple. 
Now if agent 1 needs 2-connectivity, we have

$$
\begin{gathered}
\overline{\mathcal{D}}^{1}=\left\{\omega A v_{1} C \omega ", \omega A v_{1} C B \omega^{\prime}, \omega^{\prime} B A v_{1} C \omega^{\prime \prime}\right\} \\
\Rightarrow \theta^{1}\left(\mathcal{D}^{1}\right)=1 \text { on } A v_{1} C,=\frac{1}{3} \text { on } A B C,=\frac{2}{3} \text { outside the loop }
\end{gathered}
$$

Example 2: complete graph and simple connectivity. Fix four nodes $X, Y, Z, T$ and assume all six edges are provided as illustrated by the figure below. Ann wants to connect node $X$ to node $Y$. Thus items are edges of the complete graph, and service is achieved by any path connecting the two desired nodes.

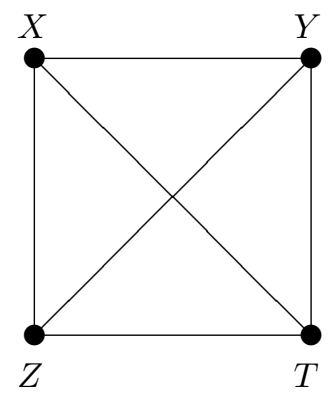

Ann's minimal serving sets are

$$
\{X Y\},\{X Z, Z Y\},\{X T, T Y\},\{X Z, Z T, T Y\},\{X T, T Z, Z Y\}
$$

therefore

$$
\theta^{1}(X Y)=\frac{1}{5}, \theta^{1}=\frac{2}{5} \text { for all other edges }
$$

Therefore if Bob needs to connect $Z$ and $T$, the counting rule charges $\frac{2}{3}$ of the cost of $X Y$ to Bob, and $\frac{1}{3}$ to Ann: a somewhat paradoxical outcome as the edge $X Y$ alone serves Ann's need but not Bob's.

The probabilistic index seems more compelling here,

$$
\theta^{p}(X Y)=\frac{1}{2}, \theta^{p}(Z T)=\frac{13}{90}, \theta^{p}=\frac{1}{4} \text { for all other edges. }
$$

It is not hard to evaluate the recommendation of the counting rule for any number of nodes and the complete graph of edges, and to show that if Ann wants to connect two nodes $X, Y$, she pays much less for the edge $X Y$ than an agent with disjoint needs.

Example 3: complete graph and 2-connectivity. On the same graph as in the previous example, Ann wants to connect nodes $X$ and $Y$ by two edgedisjoint paths. Thus her minimal serving sets are:

$$
(X Y, X Z, Z Y),(X Y, X T, T Y),(X Y, X Z, Z T, T Y)
$$




$$
(X Y, X T, T Z, Z Y),(X Z, Z Y, X T, T Y) .
$$

Hence, her counting index is now

$$
\theta^{1}(X Y)=\frac{4}{5}, \theta^{1}(e)=\frac{3}{5} \text { for } e=X Z, X T, Z Y, T Y, \theta^{1}(Z T)=\frac{2}{5}
$$

a more convincing result than in the previous example.

The probabilistic index is harder to compute, but gives the same results as the counting index except for $\theta^{p}(Z T)=\frac{224}{720}=\frac{14}{45}$.

\section{Concluding comments}

Dropping property ii) in Definition 2: Definition 2 requires that if an item is critical to agent $i$, his usage index must be strictly positive. We already noted in the discussion of Definition 2 that this property rules out the crude index $\theta\left(\mathcal{D}^{i}, a\right)=1$ if $a \in G^{i}, \theta\left(\mathcal{D}^{i}, a\right)=0$ otherwise. In some contexts, one's responsibility for an indispensable item can be regarded as intrinsically stronger than for a critical yet dispensable item: this makes property $i i$ ) in Definition 2 undesirable.

It is possible to analyze the consequences of ANO, NEU,CSY,IAN, and REP, in the absence of this requirement. We fix $R$ and $a$, and use the notation and assumptions in Step 2 of the proof of our Theorem. We use the two-person indices $\xi^{2}\left(\mathcal{D}^{1} ; \mathcal{D}^{2}\right)$ to define a binary relation $\succeq$, with strict component $\succ$, on the set $\mathcal{S C}$ of service constraints $\mathcal{D}$ :

$$
\begin{aligned}
& \mathcal{D} \succ \mathcal{D}^{\prime} \stackrel{\text { def }}{\Leftrightarrow} \xi^{2}\left(\mathcal{D}^{\prime} ; \mathcal{D}\right)=0 \\
& \mathcal{D} \succeq \mathcal{D}^{\prime} \stackrel{\text { def }}{\Leftrightarrow} \xi^{2}\left(\mathcal{D} ; \mathcal{D}^{\prime}\right)>0
\end{aligned}
$$

The relation $\succeq$ is complete because $\xi^{2}\left(\mathcal{D}^{\prime} ; \mathcal{D}\right)+\xi^{2}\left(\mathcal{D} ; \mathcal{D}^{\prime}\right)=1$, and from equation (7) (CSY) it is easy to see that it is transitive as well. ${ }^{5}$

Thus $\succeq$ orders $\mathcal{S C}$ in finitely many indifference classes $\mathcal{S C}^{k}$ : by property $i$ ) in Definition 2, the top class containing all $\mathcal{D} \in \mathcal{S C}$ for which $a$ is indispensable; by property iii) the bottom class contains all $\mathcal{D} \in \mathcal{S C}$ for which $a$ is not critical. The case covered in the theorem corresponds to two indifference classes, on one hand all $\mathcal{D}$ for which $a$ is critical, on the other hand all $\mathcal{D}$ for which it is not.

Inside each indifference class we have $\xi^{n}\left(\mathcal{D} ; \mathcal{D}^{1}, \cdots, \mathcal{D}^{n-1}\right)>0$, and if the class contains three or more elements, we find the proportional form (2), where the individual index $\theta$ is class-specific. Given an arbitrary profile $\left(\mathcal{D}^{i}, i \in N\right)$ of service constraints, the only agents $j$ with positive shares are those such that $\mathcal{D}^{j}$ is in the largest indifference class represented in $\left(\mathcal{D}^{i}, i \in N\right)$; and those agents share the cost of $a$ in proportion to the individual indices $\theta$ for that class.

\footnotetext{
${ }^{5}$ Assume $\xi^{2}\left(\mathcal{D}^{1} ; \mathcal{D}^{2}\right)>0 ; \xi^{2}\left(\mathcal{D}^{2} ; \mathcal{D}^{3}\right)>0$. If $\xi^{3}\left(\mathcal{D}^{2} ; \mathcal{D}^{1}, \mathcal{D}^{3}\right)=1$ we get a contradiction of $\xi^{2}\left(\mathcal{D}^{1} ; \mathcal{D}^{2}\right)>0$ by applying $(7)$ with $i=3$; if $\xi^{3}\left(\mathcal{D}^{1} ; \mathcal{D}^{2}, \mathcal{D}^{3}\right)=0$ we get a contradiction of $\xi^{2}\left(\mathcal{D}^{2} ; \mathcal{D}^{3}\right)>0$ by applying (7) with $i=1$. Then applying (7) with $i=2$ gives $\xi^{3}\left(\mathcal{D}^{1} ; \mathcal{D}^{2}, \mathcal{D}^{3}\right)=\left(1-\xi^{3}\left(\mathcal{D}^{2} ; \mathcal{D}^{1}, \mathcal{D}^{3}\right)\right) \cdot \xi^{2}\left(\mathcal{D}^{1} ; \mathcal{D}^{3}\right)$, and the desired conclusion $\xi^{2}\left(\mathcal{D}^{1} ; \mathcal{D}^{3}\right)>0$.
} 
Network reliability indices: The indices identified in our Theorem capture but one out of many possible approaches to the measurement of costresponsibility for redundant items. The probabilistic index described above is another idea. Yet another, also worthy of further research, uses the classic concept of reliability of a network (Ball, 1979).

Given resources $R$, and an agent $i$ with service constraints $\mathcal{D}^{i}$, suppose $\varepsilon$ is the exogenous probability of any item (link) failure, and that failures are stochastically independent. For any $T \subseteq R$, let $r_{i}(T ; \varepsilon)$ be the reliability of $R$ for $i$, i.e., the probability that she is served when only the items in $T$ are purchased. A natural individual usage index is the marginal reliability of item $a$ :

$$
\theta^{\varepsilon}\left(\mathcal{D}^{i}, a\right)=r_{i}(R ; \varepsilon)-r_{i}(R \backslash\{a\} ; \varepsilon)
$$

In the same spirit another candidate is the Shapley value of item $a$ in the cooperative game $T \rightarrow r_{i}(T ; \varepsilon)$.

\section{References}

[1] Ball, M.O. (1979), Computing network reliability, Operations Research, 27, 832-838.

[2] Holler, M. and G. Owen (2001), Power Indices and Coalition Fomation, Kluwer.

[3] Jackson, M. (2008), Social and Economic Networks, Princeton University Press.

[4] Littlechild, S.C., and G.F. Thompson (1977), Aircraft landing fees: a game theoretic approach, Bell Journal of Economics, 8, 186-204.

[5] Moulin, H. (2002), Axiomatic cost and surplus sharing, Ch. 6, in Handbook of Social Choice and Welfare (Vol. 1), Edited by Arrow, Sen and Suzumura, Elsevier.

[6] Moulin, H. (2011), Cost sharing in networks: some open questions, International Game Theory Review, to appear.

[7] Moulin, H. and F. Laigret (2011), Equal-need sharing of a network under connectivity contraints, Games and Economic Behavior, 72, 314-320.

[8] Peleg, B. and P. Sudhölter (2003), Introduction to the Theory of Cooperative Games, Springer.

[9] Shapley, L. (1953), A value for n-person games, Annals of Mathematics Study, 28, 307-318.

[10] Thomson, W. (1998), Consistency and its converse: an introduction, Working Paper, University of Rochester.

[11] Wolsey, L.A. (1998), Integer Programming, Wiley-Interscience. 